\title{
Open-source simulator for ATHENA X-ray telescope optics
}

Sironi, G.; Spiga, D.; Moretti, A.; Pareschi, G.; Tagliaferri, G.; Della Monica Ferreira, D.; Knudsen, E. Bergbäck; Jegers, A. S.; Ferreira, I.; Bavdaz, M.

\section{Published in:}

Proceedings of SPIE

Link to article, DOI:

$10.1117 / 12.2594461$

Publication date:

2021

Document Version

Publisher's PDF, also known as Version of record

Link back to DTU Orbit

Citation (APA):

Sironi, G., Spiga, D., Moretti, A., Pareschi, G., Tagliaferri, G., Della Monica Ferreira, D., Knudsen, E. B., Jegers, A. S., Ferreira, I., \& Bavdaz, M. (2021). Open-source simulator for ATHENA X-ray telescope optics. In S. L. O'Dell, J. A. Gaskin, \& G. Pareschi (Eds.), Proceedings of SPIE: Optics for EUV, X-Ray, and Gamma-Ray Astronomy $X$ [118220I] SPIE - International Society for Optical Engineering. Proceedings of SPIE - The International Society for Optical Engineering Vol. 11822 https://doi.org/10.1117/12.2594461

\section{General rights}

Copyright and moral rights for the publications made accessible in the public portal are retained by the authors and/or other copyright owners and it is a condition of accessing publications that users recognise and abide by the legal requirements associated with these rights.

- Users may download and print one copy of any publication from the public portal for the purpose of private study or research.

- You may not further distribute the material or use it for any profit-making activity or commercial gain

- You may freely distribute the URL identifying the publication in the public portal 


\title{
Open-source simulator for ATHENA X-ray telescope optics
}

\author{
INAF team: G. Sironi, ${ }^{1}$ D. Spiga, ${ }^{1}$ A. Moretti, ${ }^{1}$ G. Pareschi, ${ }^{1}$ G. Tagliaferri ${ }^{1}$ \\ DTU team: D. Della Monica Ferreira, ${ }^{2}$ E. Bergbäck Knudsen, ${ }^{3}$ A. S Jegers ${ }^{2}$ \\ ESA team: I. Ferreira, ${ }^{4}$ M. Bavdaz, ${ }^{4}$ \\ ${ }^{1}$ INAF - Brera Astronomical Observatory, Via Bianchi 46, 23807, Merate (Italy) \\ ${ }^{2}$ DTU Space, Techn. Univ. of Denmark, Elektrovej, bygn. 327, 2800 Kgs. Lyngby (Denmark) \\ ${ }^{3}$ DTU Fysik, Techn. Univ. of Denmark, Fysikvej, bygn. 311, 2800 Kgs. Lyngby (Denmark) \\ ${ }^{4}$ European Space Agency, ESTEC, Keplerlaan 1, 2201 AZ Noordwjik (The Netherlands)
}

\begin{abstract}
The ATHENA (Advanced Telescope for High Energy Astrophysics) X-ray observatory is the European Space Agency selected L2 class mission, with launch scheduled in early 2030s. The observatory hosts a large X-ray telescope designed to have 5 arcseconds resolution with an effective area larger than $1.4 \mathrm{~m}^{2}$ at $1 \mathrm{keV}$. To meet these performance requirements ESA developed the Silicon Pore Optics technology: ribbed Si plates are shaped on a proper mould to copy the defined optical design and then stacked into modules. This technological solution, taking advantage of both replica process and modular implementation, is effective to populate ATHENA's large aperture (diameter of $\sim 2.5 \mathrm{~m}$ ). As a result the optical pupil of an SPO will be very different than the classical nested shell one since it would be composed by a high number of small channels (about $10^{6}$ channels of $\sim 1 \mathrm{~mm}^{2}$ in ATHENA current design) and hence requires specific tool to be studied.

To this end ESA financed the SIMPOSIuM project aimed to develop an open source, user-friendly SPOs simulation tool. The project is now at a good level of maturity and it offers 2 Graphical User Interfaces implementing a variety of simulation features. The SPORT GUI manages a full ray-tracing code and an analytical effective area calculator. The SWORDS GUI runs a SPOs diffraction effects simulator. In this paper we present the SImPOSIuM package and its collocation in ATHENA optics development framework.
\end{abstract}

Keywords: ATHENA, silicon pore optics, optical performance, simulation

\section{INTRODUCTION}

The Advanced Telescope for High Energy Astrophysics (ATHENA) [1] was selected for the second Large Mission (L2) opportunity by the European Space Agency (ESA) in 2014 satisfying the Hot and Energetic Universe [2] theme of the Cosmic Vision. ATHENA is currently in B1 Phase with adoption foreseen for 2022 and launch due for the early 2030s. The ATHENA mission concept is based on a high throughput X-ray telescope serving two instruments: the X-ray Integral Field Unit (X-IFU) [3] a cryogenic imaging spectrometer working in the energy range $0.3-10 \mathrm{KeV}$ with unprecedented energy resolution and the Wide Field Imager (WFI) [4] working in the range $0.1-12 \mathrm{keV}$. To properly serve these instruments and fulfill the scientific requirements the mirror assembly shall have an effective area of at least $1.4 \mathrm{~m}^{2}$ with an angular resolution (Half Energy Width) smaller than 5 arcseconds and Field of View (FoV) of 40 minuts of arc. To implement an $X$ ray telescope with these characteristics a pseudo Wolter-Schwartzchild [5] configuration based on the concept of quasi-cylindrical grazing-incident nested optical surfaces in double reflecting configuration was defined. Furthermore to pack the highest possible number of nested optical surfaces in the smallest possible volume respecting the mass budget the innovative Silicon Pore Optics (SPO) technology was developed by ESA and Cosine and tested in the last decade [6], [7], [8].

${ }^{1}$ contact author: Giorgia Sironi, email: giorgia.sironi@brera.inaf.it, phone: +39-02-72320468

Optics for EUV, X-Ray, and Gamma-Ray Astronomy X, edited by Stephen L. O'Dell,

Jessica A. Gaskin, Giovanni Pareschi, Proc. of SPIE Vol. 11822, 118220I · (c)

2021 SPIE · CCC code: 0277-786X/21/\$21 - doi: 10.1117/12.2594461 

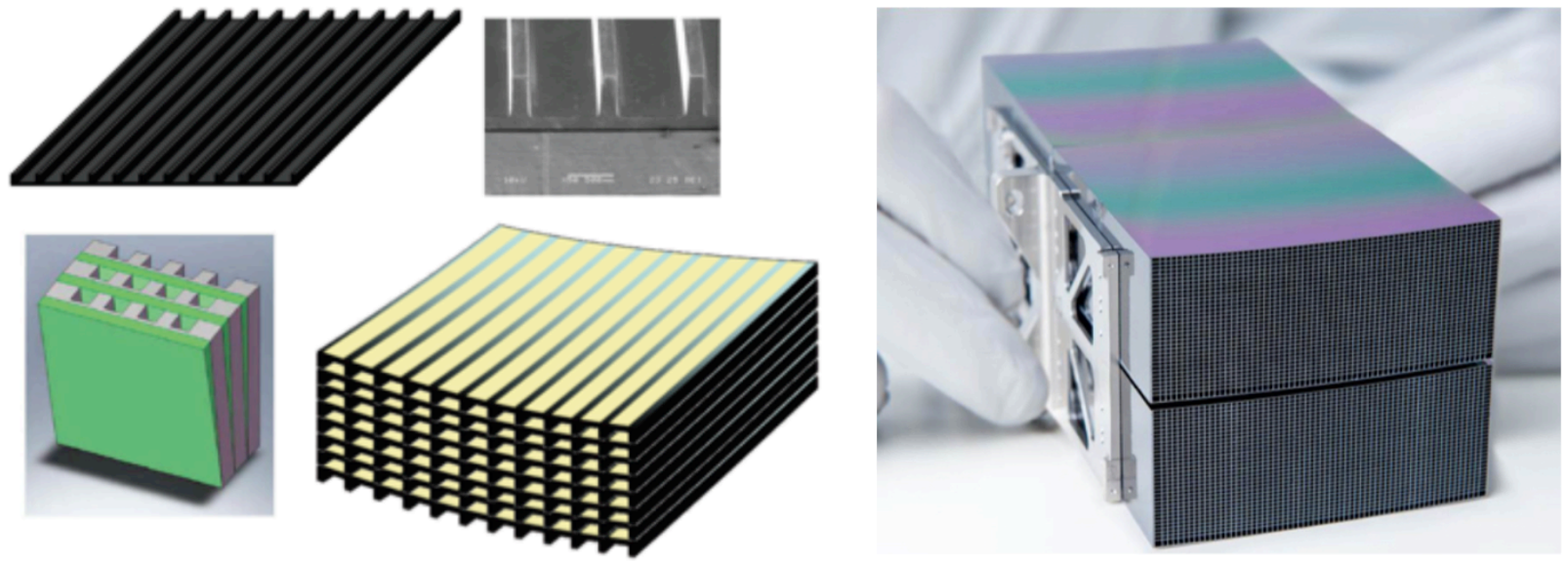

Figure 1- Manufacturing of a SPO MM: A Si wafer is diced, wedged, and ribbed. The stacking of a number of ribbed plates returns a XOU. The assembly of 4 XOUs ( 2 radially spaced primary XOUs and 2 secondary XOUs) composes a MM.

SPO are based on grooving channels (pores), bending and assembly of commercially available Si wafer (plates) already having a suitable roughness for X-ray observation purposes. This is a great advantage with respect to traditional techniques requiring that each single shell or each mandrel (if replica by electroforming is considered) is super polished to reach the micro-roughness requirement with super polishing representing the longest process in production. On the other side the use of commercially available Si wafers as optical unitary elements set a limit on the X-ray Optical Units (XOUs) maximum dimension (i. e. lateral side smaller than $12 \mathrm{~cm}$ ) with consequent enhancing of the involved XOUs' number. The ATHENA current design is based on the use of about 600 Mirror Modules (MMs) arranged in 15 rings and in 6 identical petals. Each MMs is composed by 4 XOUs, a set of 2 XOUs composing the primary reflecting surface and a set of 2 XOUs composing the second one with each XOU made up in a the stack of 38 plates bended on a mandrel (main steps of the MMs' manufacturing process are shown in Figure 1). In summary we can say that the Mirror Assembly (MA) is based on different levels of modularity and this set a strong requirement (of few percent in terms off effective area and fractions of arcseconds in terms of HEW) on the capability to simulate the optical performances of each one of the involved subsystems.

\section{THE SIMPOSIUM PROJECT}

The SIlicon Pore Optics SImulations (SImPOSIuM) project [9], [10], [11] has been funded by ESA to develop an opensource user-friendly SPO simulator with the aim to be the baseline simulator for the ATHENA optics. The project involves teams from the Istituto Nazionale di Astrofisica-Osservatorio Astronomico di Brera (INAF-OAB) and from the Danmarks Tekniske Universitet (DTU).

SImPOSIuM is now running since 5 years and the first release of the produced SW tools to the scientific community was held on spring 2021. The project will be completed within 2022 with the implementation of the beta-testers feedbacks.

The main challenge of the SIMPOSIUM project is the accurate simulation of the optical performances of a million of SPO of the ATHENA MA. Moreover, SIMPOSIUM is supporting the development of the ATHENA optics in still open design issues, integration tolerances settings and calibration facilities development. Hence it was/is fundamental to interact with the scientific community to define the features required by the tools. SIMPOSIUM is currently supporting the following activities:

- Design: arrived in phase B1 the design of ATHENA'a MA is almost frozen, but a number of details strongly impacting on the final optical performances are still to be defined. The definition of these aspects are currently driven by manufacturing feasibility limits but shall be evaluated on the basis of simulated results. 
- Plates: the plates are diced with a wedged cut from Si wafers and then the channels representing the optical pores are grooved on them. The groves dimension is obviously the minimum optical unit. Larger pores would for sure increase the effective area but would also lead to optical degradation due to straylight. The wedging angle represents the change in the incidence angle of the optical surface and shall be chosen as the best approximation for the incidence angles theoretically changing from plate to plate through the whole XOU. The capability to simulate different wedging angles and pore dimension was then implemented in SIMPOSIUM.

- XOUs: each XOU is the result of the stacking of $38 \mathrm{Si}$ plates (36 effectively used as optical surfaces) on a mandrel. Note that the accuracy in copying the mandrel's shape drops moving away from the mandrel itself and that the only measured surface would be the last one, while the PSF of each single MMs will be measured at the calibration facilities. The capability to reproduce the measured PSF accounting for the plates shape error evolution through the XOU is hence very important and under development.

- MMs: MMs are formed by a primary and secondary reflecting XOUs, to simplify the manufacturing the current design foresees a conical primary surface and a secondary one optimized to take under control the optical degradation across the FOV. The capability to simulate the optical performance of the MMs considering the reciprocal primary and secondary surfaces distance, angles and profile is then necessary to define the details of the second reflecting surface for each ring.

- Integration: the integration process of ATHENA's MA will be held by companies developing specific facilities and tools. To estimate the tolerances on the integration tools it is necessary to be able to evaluate the possible misalignment, gravity and thermal effects of the complete MA support structure. SIMPOSIUM is supporting the integration facilities needs by implementing:

O the capability to simulate the diffraction effect of UV-lights in SPO: the single MMs will be aligned at an UV optical bench with the aim to produce the best common focus in the X-rays. A tool capable to simulate what is expected in the X-rays for ab optical response measured in UV is then needed;

o the capability to load measured or simulated reciprocal MMs misalignments: the tolerances on the integration and mechanical tools can be set only by the simulation of the optical degradation produced by the possible errors. Hence SIMPOSIUM was equipped with the capability to use misalignment tables indicating each MM position and orientation.

- Calibration: The calibration of the ATHENA's MA will be obtained in steps. The calibration of single MMs at 2 energies (1.5 and $4.5 \mathrm{keV}$ ) with a collimated beam (with a divergence smaller than the MMs one) are planned to be performed at a facility like the BEATRIX [12] in construction at INAF-OAB. The calibration of the full MA is planned at the VERT-X calibration facility [13] and at the upgraded PANTER facility [14].

For the requirement setting of all these facilities it is necessary to simulate the behavior of the optics under specific conditions like the source distance and dimension, system's perturbations caused by the dynamical errors of the facilities' alignment tools, possible masked illumination etc. SIMPOSIUM is supporting these activities and collecting specific request from the ATHENA calibration working group.

- Scientific data simulators: simulation tools play an important role to predict the expected performance of astronomical instrumentation. Specific simulators capable to reproduce in detail the detector response and the astronomical source input were developed in the last decades for various X-ray missions. The tool adopted by the scientific community to simulate the ATHENA's instruments response is SIXTE (Simulator for X-ray Telescopes) [15]. SIXTE is specifically focused to take into account the detector effects for different kind of complex sources (extended sources, bright sources causing pile-up, timing analysis of variable sources). On the other hand SIXTE is not simulating the optical performance but taking a PSF (simulated or measured) as input. Hence to have an efficient simulation chain the output of SIMPOSIUM was planned to be exportable in the SIXTE optical response input format. 


\section{THE SIMPOSIUM TOOLS}

SIMPOSIUM tools are organized in two graphical user interfaces: i) SPORT (Silicon Pore Optics Ray-Tracer) implementing the McXTrace [16] Montecarlo ray-tracer for X-ray optics and an effective area analytical calculator and ii) SWORDS (Silicon-wise Optic Rapid Diffraction Simulator) a SPOs MMs diffraction simulator. Both GUIs allows the upload of a common input file to provide the current ATHENA design.

\subsection{SPORT}

The SPORT GUI allows the user to define a SPO X-ray telescope configuration, set an experimental configuration and compute the optical response in terms or PSF and effective area. The software is very flexible and implements the tools to simulate all the perturbations previously listed. SPORT is an open source tool for Mac OS, Windows and Linux operative systems. The installation package, the manual and the updates can be downloaded on-line.

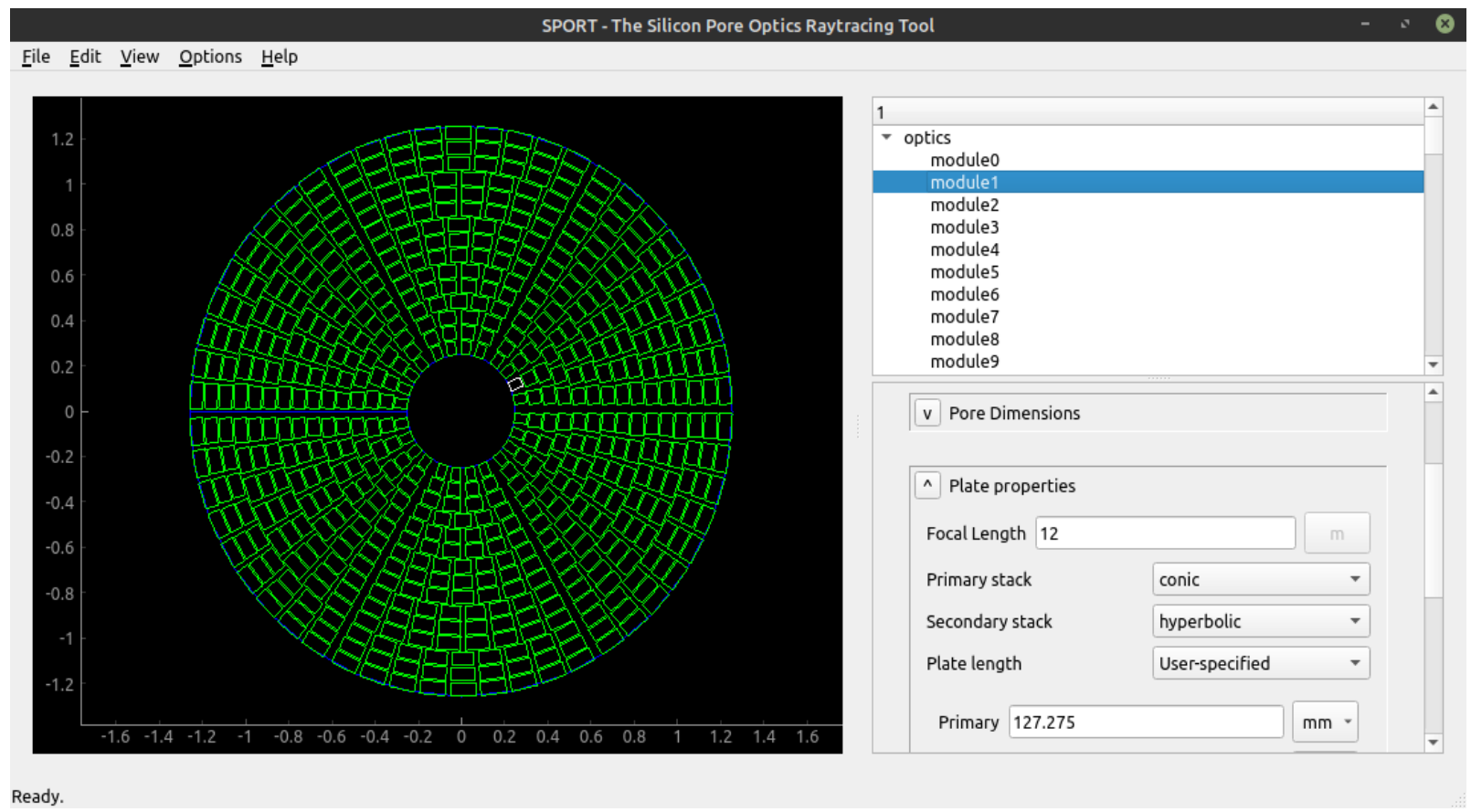

Figure 2 - The optics editor of SPORT showing the ATHENA current MMs layout organized in 6 identical petals and 15 rings. In the right part of the editor is shown the hierarchical organization of the MMs definition where in the plate tab the user can define the focal length and the configuration (conical primary surface and hyperbolic second one) for the MM's plates.

A full description of the input for SPORT is available in the manual at the downloading page but we summarize here its main features:

- Optics editor: the optics editor (Figure 2) allows to set a new SPO based X-ray telescope configuration organized on hierarchical levels of petals, rings, modules, plates and pores or to upload a predefined configuration. In this section is also possible to load different reflectivity tables (simulated or measured) for reflecting pores' surface, back planes and ribs. In the same way it is possible to enable the roughness option to take into consideration non-specular reflectivity given a roughness level (the model works on pre-loaded lookup tables generated by the surface power spectral density for different energies and angles).

A file containing the ATHENA current MMs layout and baseline coating as defined by ESA is available with the SW package. 

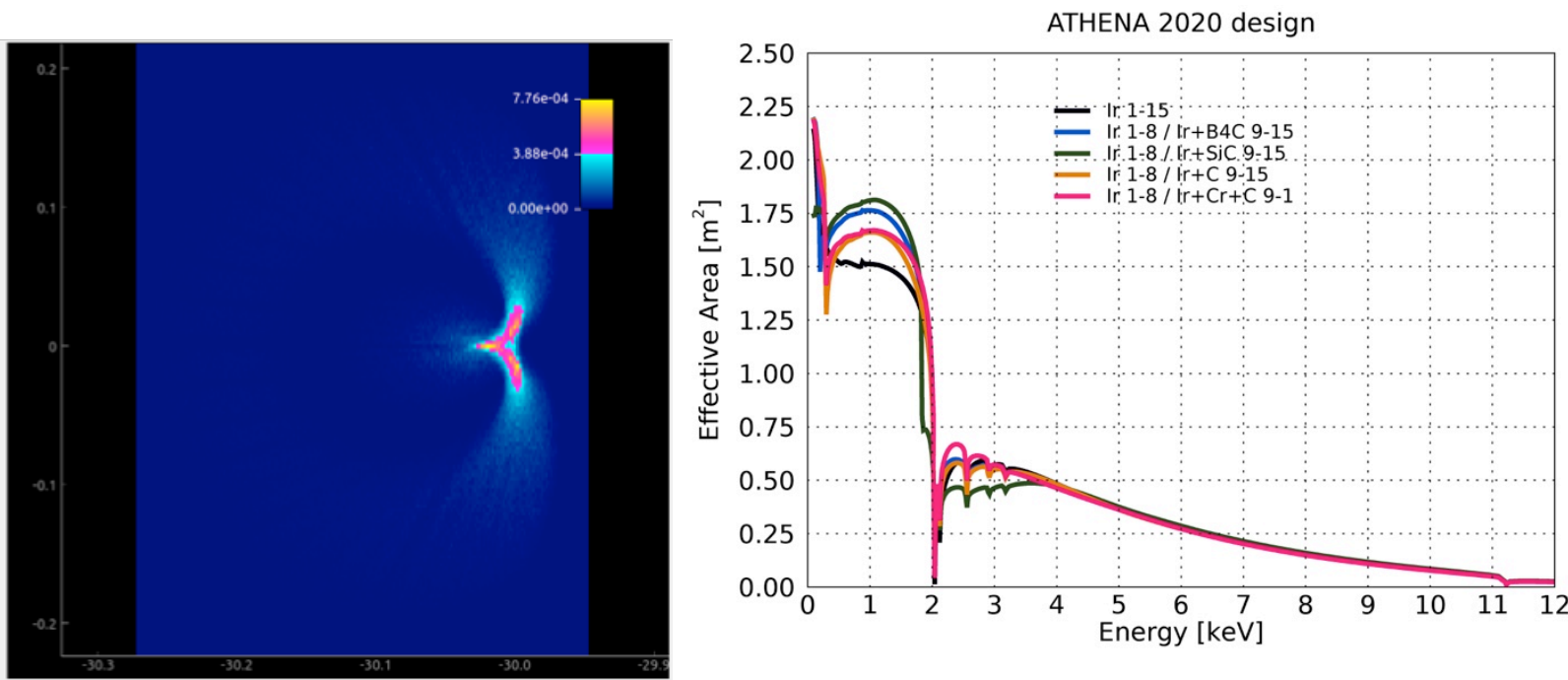

Figure 3 - Left: image of the SPORT result editor showing a PSF obtained for a point-like off-axis source. Right: effective area curve computed analytically for different coatings.

- Simulation editor: the simulation editor allows the user to set the experiment configuration, hence here are stored the source characteristics like distance, off-axis position, extension and spectrum. For extended source SPORT offers also the opportunity to load a .FITS file with a complete map with dimension comparable to the FOV. In this section the user can also set the number of the considered photons and the number and position (focal plane, intra or extra focal) at which collect the image.

- Results editor: the results editor allows to show the heat map or the effective area graph of the photon collected at the defined focal distance. The results can be visualized for the complete set of photons or for sub-sets that can be selected defining filters. Filters can be: i) spatial to mask partially the MM, ii) source to define when multiple sources are considered or iii) defined by the optical path of the photons reaching the screen plate at the focal distance. This last filter in particular permits to discriminate and visualize separately photons that underwent single reflection (on primary or secondary surfaces), double reflection or no reflection at all allowing the study of stray-light effects.

An example of the results obtained for the simulation of the current ATHENA layout for a point like and for an extended source as a function of the optical configuration approximation (conical/conical, conical/hyperbolic, parabolic/conical, Wolter) is given in [17].

A view of the results editor is shown in Figure 3: on the left we report an example of the PSF obtained for a point-like off-axis source while on the right we report the effective area curve computed analytically for different coatings.

\subsection{SWORDS}

SWOrDS is the GUI realized to run the SPO diffraction simulator. SWOrDS allows the user to simulate the PSF of a MM by means of physical optics taking into account the reflecting surfaces figure errors and the diffraction effects due to the small size of the pores. The code is based on the methods described in [9] and [10] and is developed under the IDL 8.6 environment. The SWOrDS GUI is developed under MacOS environment and XQuartz graphical interface.

The SWORDS GUI visualize a main-frame (Figure 4) giving access to different menus for input configuration:

- SPO configuration menu: here the parameters defining the MMs optical configuration are set (pore dimension, plate number, focal length, optical surface profile and wedge). 
- Shape error menu: in this menu all the perturbations to perfect modeled optics can be managed. SWORDS offers the possibility to insert roto-translations errors between primary and secondary stacks, to define sagittal errors as sinusoids/polynomials or to impose a Lorentian HEW response and to define the errors of the radius of curvature.

- Setup menu: in this menu it is possible to set the experimental configuration of the light source and of the detector. The light source can be monochromatic or with an energy bandwidth extension and its distance, offaxis angle and extension shall be set. For what concerns the detector the dimension, resolution and distance from the MM have to be set.

Once all the input steps are concluded and the set up configuration defined SWORDS allows the user to compute the Complex Pupil Function (CPF) and to visualize it in heat map and finally to compute the diffraction pattern at the detector.

As an example of the use of the diffraction simulator we report the result of the diffraction pattern obtained by a MM in wedging configuration $-1 /+1$ when illuminated in X-rays $(40 \mathrm{~A})$ and un UV-light (1000 A). The images shown in Figure 5 are scaled in wavelength dimension; hence the right panel has a side dimension that is 10 times the dimension of the left one. As can be seen from the figure the dimension of the PSF in X-rays (that is of about 0.7 arcseconds) is not reproducible in UV-light. This means that a UV integration facility shall work on MMs barycenter alignments but won't be able to directly measure the MMs HEW.

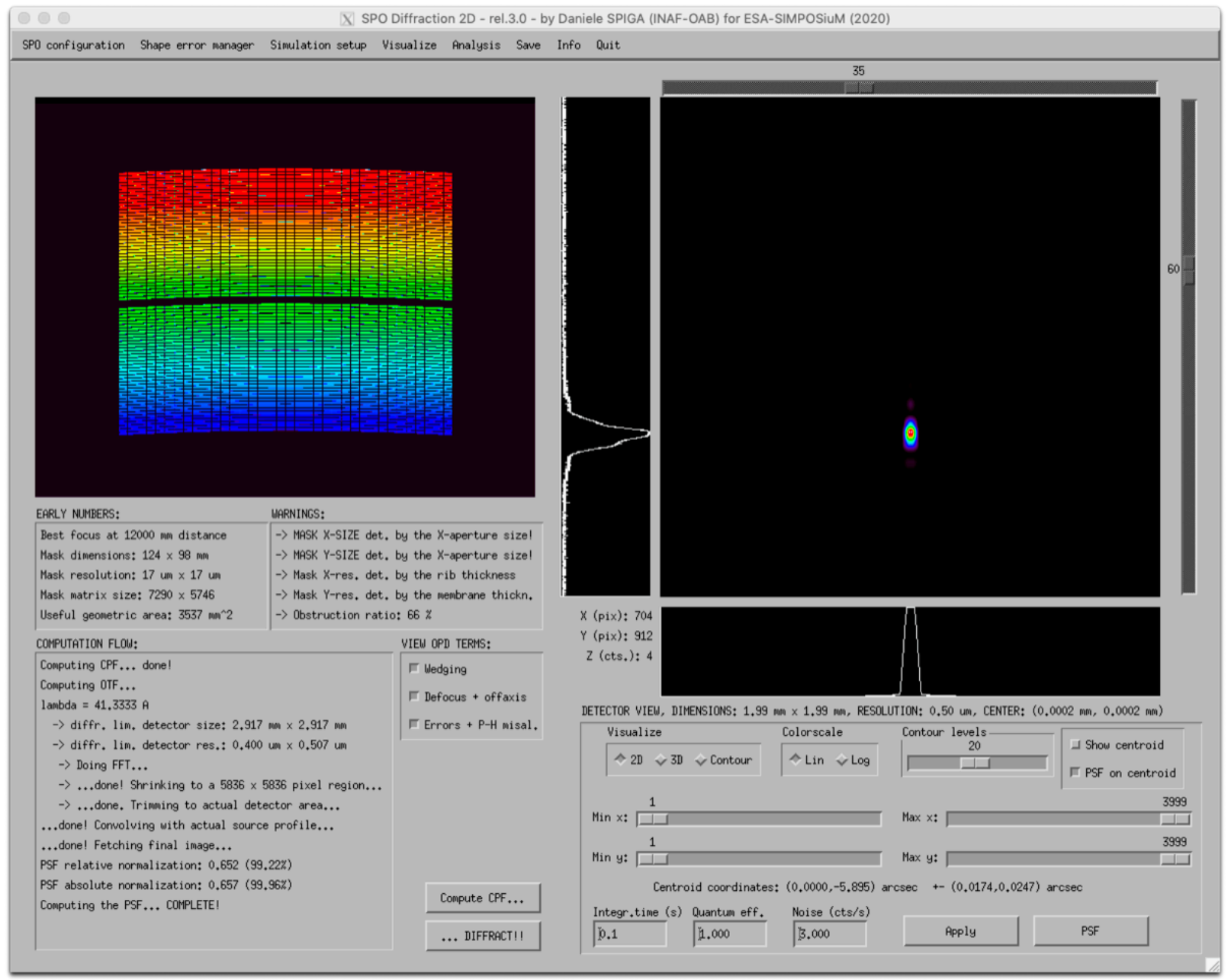

Figure 4 - The SWORDS main-frame showing the computed CPF heat map and the PSF at the detector. 

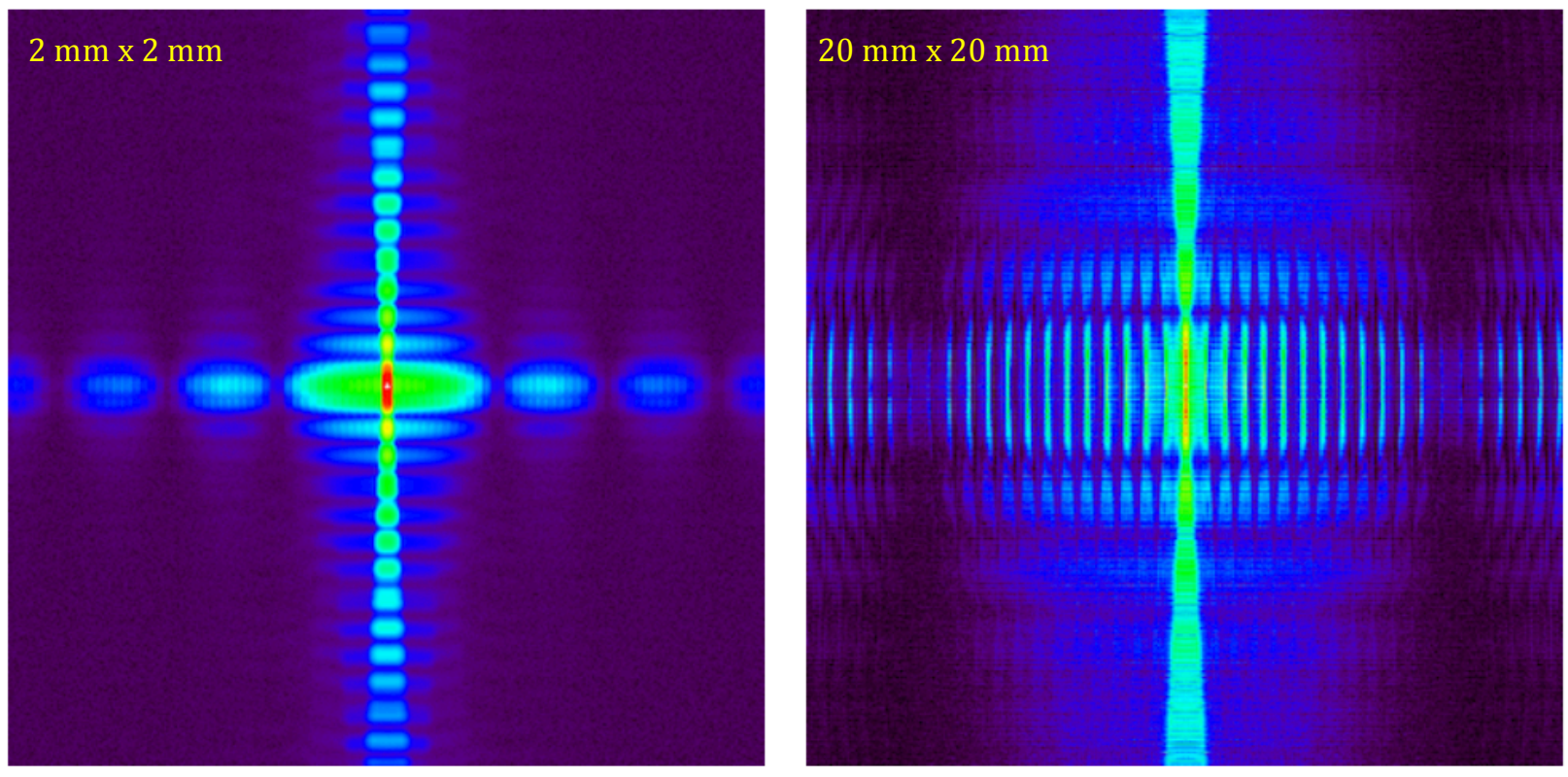

Figure 5 - (left) the focal spot at $\stackrel{\circ}{1}=40 \AA, 2 \mathrm{~mm}$ field, (right) at $1=1000 \AA, 20 \mathrm{~mm}$ field. Log color scale.

\section{CONCLUSIONS}

SIMPOSIUM is a project funded by ESA and developed by INAF-OAB and DTU for the development of an open source simulator to simulate the optical performances of SPO based X-ray telescope. The project aims to develop an open-source, user-friendly ATHENA optics simulator to be used as baseline tool in the framework of the ATHENA mission development.

The project is running since 5 years and is at a good level of maturity. The SIMPOSIUM tools are organized in two GUIs: i) SPORT implementing a ray-tracing and a fast effective area analytical calculator and ii) SWORDS dedicated to the diffraction pattern computation of MMs. Both tools have the aim to implement the ESA design with the highest possible level of accuracy and to offer outputs for the scientific simulations and for the evaluation of the accuracies needed by the integration/calibration facilities.

The SIMPOSIUM's tools are open source and currently in beta-testing phase. A final release, implementing the scientific community's feedback, will be ready in spring 2022.

We encourage the use of the package by all relevant people involved in the ATHENA development to allow us to implement in the best way the simulation needs of the community. Please contact us if you want to download the first release of the software, with the manuals and the ATHENA current baseline configuration files.

\section{REFERENCES}

[1] Nandra, K., Barret, D., Barcons, X., et al., "The Hot and Energetic Universe: A White Paper presenting the science theme motivating the Athena mission", http://arxiv.org/abs/1306.2307 (2013)

[2] "ATHENA: The Advanced Telescope for High Energy Astrophysics: A mission addressing The Hot and Energetic Universe science theme", http://sci.es.int/jump.cfm?oid=52030 (2014)

[3] Barret, D. and 100 colleagues “The ATHENA X-ray Integral Field Unit (X-IFU)” Proc. SPIE 10699. 
doi:10.1117/12.2312409 (2018)

[4] Meidinger, N., "The wide field imager instrument for Athena”, Proc. SPIE 9905. doi:10.1117/12.2231604 (2016)

[5] Van Speybroeck, L.P., Chase, R. C., "Design parameters of paraboloid-hyperboloid telescopes for X-ray astronomy," Appl. Opt. 11(2), 440 (1972)

[6] Bavdaz, M., Wille, E., Shortt, B., et al., "The ATHENA optics development," Proc. SPIE 9905, 990527 (2016)

[7] Collon, M. J., Vacanti, G., Gu nther, R., et al., "Silicon pore optics for the ATHENA telescope," Proc. SPIE 9905, 990528 (2016)

[8] Collon, M., Vacanti, G., Barri'ere, N., et al., "Development of Athena mirror modules," Proc. SPIE 10399, 103990C (2017)

[9] Spiga, D., Christensen, F. E., Bavdaz, M., et al., "Simulation and modeling of silicon pore optics for the ATHENA x-ray telescope," Proc. of the SPIE, Vol. 9905, 990550 (2016)

[10] Spiga, D., Della Monica Ferreira, D., Shortt, B., et al., "Optical simulations for design, alignment, and performance prediction of silicon pore optics for the ATHENA x-ray telescope," Proc. of the SPIE, Vol. 10399, 103990H (2017)

[11] Sironi, G., Spiga, D., Della Monica Ferreira, D., et al., "Simulating the optical performances of the ATHENA xray telescope optics," Proc. of the SPIE, Vol. 10699, 103993N (2018)

[12] Spiga, D., Pelliciari, C., Bonnini, E., et al., "An expanded x-ray beam facility (BEaTriX) to test the modular elements of the ATHENA optics," Proc. SPIE 9144, 91445I. doi:10.1117/12.2057358 (2014)

[13] Moretti, A., Pareschi, G., Uslenghi, M., et al. "VERT-X: VERTical X-ray raster-scan facility for ATHENA calibration. The concept design," Proc. SPIE 11119, 111190O. doi:10.1117/12.2530713 (2019)

[14] Burwitz, V. and 19 colleagues "X-ray testing at PANTER of optics for the ATHENA and Arcus Missions," ICSO, 11180. doi:10.1117/12.2535995 (2018)

[15] Dauser, T., Falkner, S., Lorenz, M., et al. "SIXTE: a generic X-ray instrument simulation toolkit," AAP, 630, A66. doi:10.1051/0004-6361/201935978 (2019)

[16]E. B. Knudsen, A. Prodi, P. Willendrup, K. Lefmann, J. Baltser, C. Gundlach, M. S. del Rio, C. Ferrero, and R. Feidenhans'1, "McXtrace: a modern ray-tracing package for x-ray instrumentation," Proc. SPIE 8141, pp. 130 136, (2011).

[17] Jegers A.S., Della Monica Ferreira D. Knudsen E. B. et al,"Effect of mirror curvature on the angular resolution of Athena's optics,” Proc. SPIE 11822, , (2021). 